\title{
Beaming matter waves from a subwavelength aperture
}

\author{
A. I. Fernández-Domínguez, ${ }^{1}$ Esteban Moreno, ${ }^{1}$ L. Martín-Moreno, ${ }^{2}$ and F. J. García-Vidal ${ }^{1}$ \\ ${ }^{1}$ Departamento de Física Teórica de la Materia Condensada, Universidad Autónoma de Madrid, E-28049 Madrid, Spain \\ ${ }^{2}$ Departamento de Física de la Materia Condensada, Universidad de Zaragoza-CSIC, E-50009 Zaragoza, Spain
}

(Received 5 May 2006; published 17 August 2006)

\begin{abstract}
We show theoretically that the shape of a beam of matter waves (cold atoms) emerging from one subwavelength aperture pierced in a film can be collimated within a few degrees. By means of an external laser field, a potential well for the atoms in the direction perpendicular to the surface is created. In this way, a running surface matter wave can be excited when atoms diffract from the aperture. If the aperture is surrounded with a finite array of indentations, coherent scattering of the surface matter wave with these indentations molds the angular distribution of the matter wave in the far field.
\end{abstract}

DOI: 10.1103/PhysRevA.74.021601

PACS number(s): 03.75.Be, 32.80.Lg, 42.50.Vk, 73.20.Mf

Together with the understanding of the atom-surface interactions [1], one of the main goals in atom optics is to control the flow of matter waves through various structures [2]. The proper design of such structures could open up the possibility of building devices fulfilling simple functionalities. Trapping and guiding matter waves are the basic ingredients and there are several schemes to achieve these effects [3]. Another essential building block to control cold atoms is the coupling from free matter waves to confined (e.g., trapped or guided) matter waves. The knowledge gained in other research areas such as optics and plasmonics can help us in the design of the mentioned devices.

For the case of photons, it has recently been demonstrated that surface electromagnetic (EM) waves constitute a route to mold the flow of light. Extraordinary transmission (ET) of light through a periodic two-dimensional (2D) array of subwavelength holes perforated on a thick metallic film has been reported [4], and there have been several works showing the same ET effect on a single subwavelength aperture surrounded by periodic corrugations [5]. Moreover, it has been also shown how the light emerging from a subwavelength aperture can be collimated within a few degrees by corrugating the output surface of the hole with a finite array of indentations [5]. The physical origin of both ET and beaming phenomena stems from the excitation of surface EM modes (surface plasmons) on the corrugated metal-dielectric interfaces [6-8].

In a very recent paper, we have theoretically demonstrated that the ET phenomenon could also appear for matter waves [9]. We showed how $100 \%$ transmission of rubidium atoms through a periodic 1D array of slits much narrower than the atomic de Broglie wavelength could be attained. The main ingredient needed to observe this resonant ET phenomenon was the excitation by the incident atoms of a surface matter wave supported by the structure. The aim of the present paper is to study the appearance of the ET phenomenon for matter waves through one single subwavelength aperture, and also to address the possibility of creating collimated matter wave beams by just corrugating the output surface that surrounds the subwavelength aperture. As we will show, the main advantage of the approach we propose is that the crosssection diameter of the emerging beams is of the order of only several de Broglie wavelengths.

In Fig. 1 we show the considered structure. A single 1D slit of width $w$ perforated on a film of thickness $t$ is flanked in both input and output surfaces by a finite periodic array of $2 N$ grooves of width $w_{\mathrm{g}}$ and depth $t_{\mathrm{g}}$ separated by a distance $\Lambda$. Atoms impinge along the direction $z$ normal to the film. The corresponding 2D potential landscape for the impinging atoms is the following. The film is considered opaque to atoms and therefore the potential inside it is assumed to be infinite (black). In dark gray regions of width $h$ the potential is $-V$, and in light gray regions the potential is zero. In this way, two potential wells in the $z$ direction are constructed at the two surfaces of the film. For appropriate values of $h$ and $V$, each potential well may support a bound state with its wave function confined in the $z$ direction but able to propagate in the $x$ direction, which we name a surface matter wave (SMW). Inside the slit the value of the potential is $-V$ and inside the grooves $-V_{\mathrm{g}}$. Although this is a toy model, let us remark that a feasible implementation of similar 2D potentials has already been proposed [9]. As it is done with atomic mirrors [10], the idea is to take advantage of the attraction given by both the gravitational force and the long-range van der Waals atom-surface interaction, and add a short-range

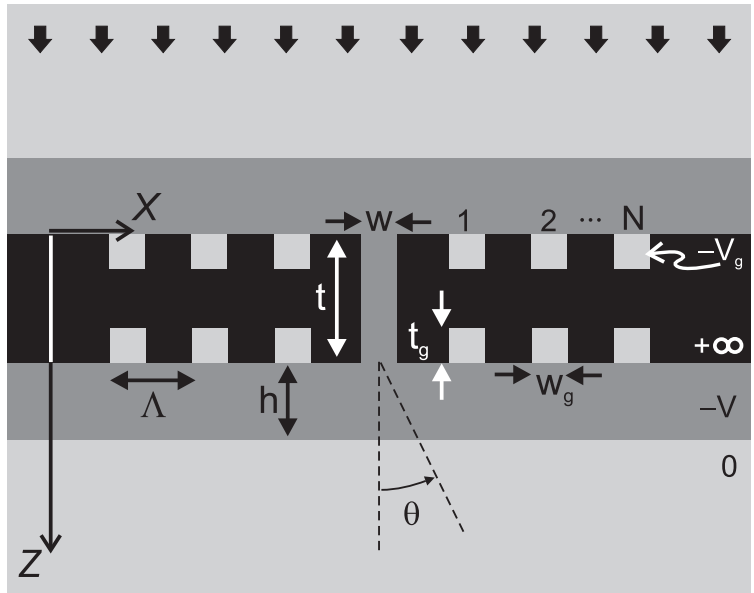

FIG. 1. Schematic picture of the analyzed structure. A single slit of width $w$ perforated on a film of thickness $t$ flanked symmetrically by $2 N$ grooves of width $w_{\mathrm{g}}$ and depth $t_{\mathrm{g}}$. The distance between consecutive grooves is $\Lambda$. Atoms are impinging from top and feeling a potential landscape described by the different shading regions (see main text). 
repulsive dipolar interaction due to a blue-detuned laser field $[11,12]$. A $2 \mathrm{D}$ potential landscape similar to the one depicted in Fig. 1 could, in principle, be created by considering a finite array of parallel cylindrical optical fibers carrying a blue-detuned optical mode. This optical mode is propagating along the fibers and evanescent in the perpendicular plane outside them. The separation between the fibers is such that only a central channel could allow the (evanescent) tunneling of atoms.

For the calculations presented in this paper we have chosen the following set of geometric parameters: $w=0.3 \mu \mathrm{m}$, $t=0.16 \mu \mathrm{m}, w_{\mathrm{g}}=0.22 \mu \mathrm{m}, t_{\mathrm{g}}=0.065 \mu \mathrm{m}, \Lambda=0.8 \mu \mathrm{m}$, and $h=0.3 \mu \mathrm{m}$. As regards the potential values, $V$ is fixed to $1.06 \times 10^{-11} \mathrm{eV}$, resulting in the existence of a bound state of energy $E_{0}=-0.095 \times 10^{-11} \mathrm{eV}$ mainly located inside the potential well. In order to analyze the dependence of the transmission properties as a function of the potential $V_{\mathrm{g}}$ inside the grooves, this quantity will be varied from $V_{\mathrm{g}}=V$ down to $V_{\mathrm{g}} \approx 19 \mathrm{~V}$. Needless to say, the ET phenomenon for matter waves would also appear for other ranges of geometric and potential parameters and our election is just for proof-ofprinciple purposes. With the chosen parameters the resonant phenomena to be described occur for atoms with kinetic energies of the order of microkelvins.

In order to compute the transmission properties of this type of structure, we apply a modal expansion of the wave function $(\Psi)$ in the different regions. The central slit and the grooves support an infinite number of eigenmodes but, as their widths are subwavelength, a good approximation is to consider only the fundamental mode inside them. Then, for $x$ inside indentation $\alpha, \Psi$ is a linear combination of $\phi_{\alpha}(x) e^{ \pm i q_{\mathrm{zg}} z}$, where $\phi_{\alpha}(x)=\langle x \mid \alpha\rangle$ $=\sqrt{2 / w_{\mathrm{g}}} \sin \left[\left(x+w_{\mathrm{g}} / 2-d_{\alpha}\right) \pi / w_{\mathrm{g}}\right]$. Here $d_{\alpha}$ measures the coordinate of indentation $\alpha$ with respect to the central slit. The propagation constant $q_{z \mathrm{~g}}$ is equal to $\sqrt{\left(1+V_{\mathrm{g}} / E\right) k_{0}^{2}-\left(\pi / w_{\mathrm{g}}\right)^{2}}$, with $E$ being the energy of the incoming matter wave with wave number $k_{0}=\sqrt{2 m E} / \hbar=2 \pi / \lambda_{\mathrm{dB}}, m$ the mass of the atoms ( $\mathrm{Rb}$ atoms are considered), and $\lambda_{\mathrm{dB}}$ their de Broglie wavelength. For $\alpha=0$ (central slit), $w_{\mathrm{g}}=w$ and $V_{\mathrm{g}}=V$. In the remaining regions (with potential $-V$ or 0 ) we expand $\Psi$ by a set of plane waves. Let us denote $\left\{\psi_{\alpha}, \psi_{\alpha}^{\prime}\right\}$ $(\alpha=-N, \ldots, N)$ the modal amplitudes of the wave function at the entrances of the indentations. The subset $\left\{\psi_{\alpha}\right\}$ determines $\Psi$ at the input surface $\Psi\left(z=0^{+}\right)=\Sigma_{\alpha} \psi_{\alpha} \phi_{\alpha}(x)$ whereas the other subset $\left\{\psi_{\alpha}^{\prime}\right\}$ gives $\Psi$ at the output surface $\Psi\left(z=t^{-}\right)=\Sigma_{\alpha} \psi_{\alpha}^{\prime} \phi_{\alpha}(x)$. Projecting the continuity equations for $\Psi$ at $z=0$ and $z=t$ onto plane waves, the reflection and transmission coefficients can be expanded in terms of the set $\left\{\psi_{\alpha}, \psi_{\alpha}^{\prime}\right\}$. Imposing the remaining continuity conditions for $\Psi$ and its derivative, a system of $4 N+2$ equations for these unknowns $\left\{\psi_{\alpha}, \psi_{\alpha}^{\prime}\right\}$ is found:

$$
\begin{aligned}
& \left(G_{\alpha \alpha}-\epsilon_{\alpha}\right) \psi_{\alpha}+\sum_{\beta \neq \alpha} G_{\alpha \beta} \psi_{\beta}-G_{V} \psi_{0}^{\prime} \delta_{\alpha 0}=I_{\alpha}, \\
& \left(G_{\alpha \alpha}-\epsilon_{\alpha}\right) \psi_{\alpha}^{\prime}+\sum_{\beta \neq \alpha} G_{\alpha \beta} \psi_{\beta}^{\prime}-G_{V} \psi_{0} \delta_{\alpha 0}=0 .
\end{aligned}
$$

In this system of linear equations, $G_{\alpha \beta}=\langle\alpha|\hat{G}| \beta\rangle$ repre-

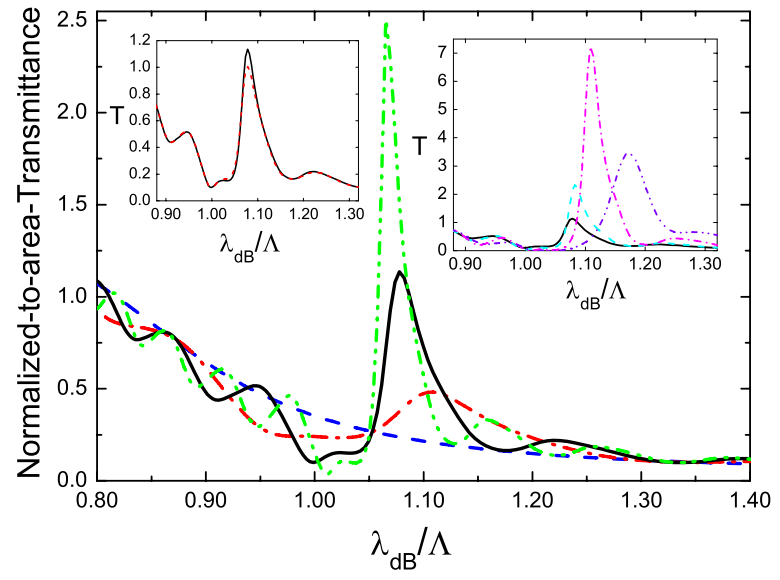

FIG. 2. (Color online) Main panel shows normalized-to-area transmittance versus $\lambda_{\mathrm{dB}}$ for four different structures. Dashed line: single slit. Dash-dotted line: single slit flanked by $N=5$ grooves located at each side (left and right) of the central slit in both (upper and lower) surfaces. Solid and dash-double-dotted lines: same as before but with $N=10$ and 15 , respectively. In these four cases $V_{\mathrm{g}}=V$. Right inset displays the dependence of $T$ on the potential inside the grooves for $N=10$. Solid line: $V_{\mathrm{g}}=V$. Dashed line: $V_{\mathrm{g}}=9.5 \mathrm{~V}$. Dash-dotted line: $V_{\mathrm{g}}=17 \mathrm{~V}$. Dash-double-dotted line: $V_{\mathrm{g}}=19 \mathrm{~V}$. Left panel renders $T$ versus $\lambda_{\mathrm{dB}}$ for $N=10$ for grooves milled at the two surfaces (solid line) or only at the input surface (dashed line).

sents the coupling between indentations $\alpha$ and $\beta$. The representation of the propagator $\hat{G}$ in real space can be written as

$$
G\left(x, x^{\prime}\right)=\left\langle x|\hat{G}| x^{\prime}\right\rangle=\frac{i}{2 \pi k_{0}} \int_{-\infty}^{\infty} d k_{x} k_{z} C\left(k_{x}, h\right) e^{i k_{x}\left|x-x^{\prime}\right|}
$$

with

$$
C\left(k_{x}, h\right)=\frac{k_{z}^{(0)} \cos k_{z} h-i k_{z} \sin k_{z} h}{k_{z} \cos k_{z} h-i k_{z}^{(0)} \sin k_{z} h},
$$

where $k_{z}^{(0)}=\sqrt{k_{0}^{2}-k_{x}^{2}}$ and $k_{z}=\sqrt{\xi^{2} k_{0}^{2}-k_{x}^{2}}$ with $\xi \equiv \sqrt{1+V / E}$. The nonhomogeneous term in Eqs. (1), $I_{\alpha}=(2 \sqrt{2} i / \pi) \xi /$ $\left[\xi \cos \left(k_{0} \xi h\right)-i \sin \left(k_{0} \xi h\right)\right]$, measures the overlap between the fundamental eigenmodes inside the indentations and the normal incident matter wave. The information about the multiple reflections of the wave inside indentation $\alpha$ is carried by $\epsilon_{\alpha}=q_{z \mathrm{~g}} /\left[k_{0} \tan \left(q_{z \mathrm{~g}} t_{\mathrm{g}}\right)\right]$, and $G_{V}=q_{z 0} /\left[k_{0} \sin \left(q_{z 0} t\right)\right]$ takes into account the coupling between both sides of the central slit. Once the set $\left\{\psi_{\alpha}, \psi_{\alpha}^{\prime}\right\}$ is obtained, the transmittance through the slit can be calculated as $T=\operatorname{Im}\left[G_{V}^{*} \psi_{0}^{*} \psi_{0}^{\prime}\right]$. As the incident wave is normalized such that the impinging flux integrated over the area of the slit is unity, $T$ corresponds to the normalized-to-area transmittance.

We now present the results obtained with this formalism, starting with the transmission enhancement through one single subwavelength aperture. Figure 2 represents $T$ versus the de Broglie wavelength $\lambda_{\mathrm{dB}}$ for normal incidence of the atoms. In this figure we analyze the dependence of the transmittance with the number of grooves surrounding the central slit. The dashed curve shows the transmittance $(T)$ for the 


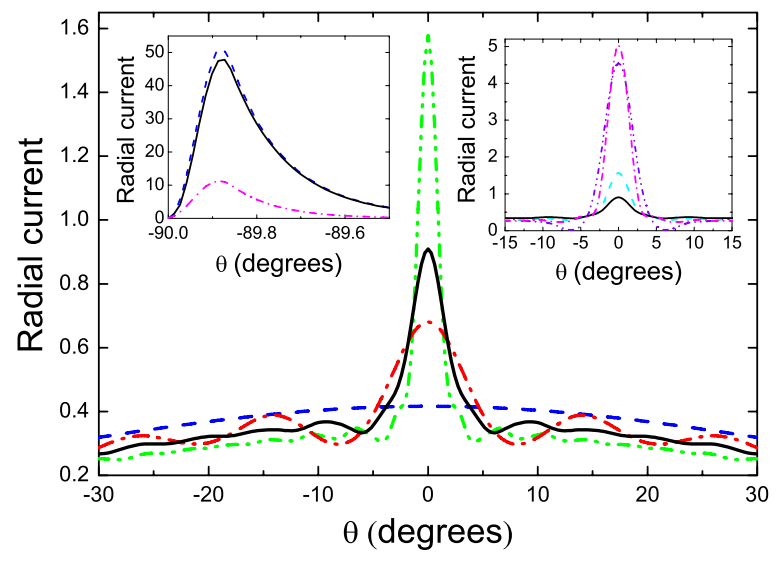

FIG. 3. (Color online) Main panel shows the radial component of the current $\left(J_{r}\right)$ versus $\theta$ for four different structures. The current is evaluated at resonance and at a distance $150 \Lambda$ from the output surface. Dashed line: single slit. Dash-dotted line: single slit flanked by $N=5$ grooves located at the left and right of the central slit. Solid and dash-double-dotted lines: same as before but with $N=10$ and 15 , respectively. In these four cases $V_{\mathrm{g}}=V$. Right inset displays the dependence of $J_{r}$ on the potential inside the grooves for $N=10$. Solid line: $V_{\mathrm{g}}=V$. Dashed line: $V_{\mathrm{g}}=9.5 V$. Dash-dotted line: $V_{\mathrm{g}}=17 \mathrm{~V}$. Dash-double-dotted line: $V_{\mathrm{g}}=19 \mathrm{~V}$. Left panel renders $J_{r}$ versus $\theta$ in the vicinity of $90^{\circ}$ for three cases. Dashed line: single slit. Solid line: $N=10$ and $V_{\mathrm{g}}=V$. Dash-dotted line: $N=10$ and $V_{\mathrm{g}}=17 \mathrm{~V}$.

single-slit case (no additional grooves). Since the energy range is such that $\lambda_{\mathrm{dB}}>2 w$, then $T<1$, presenting an exponential decay as $\lambda_{\mathrm{dB}}$ is increased. When grooves are added (located symmetrically with respect to the central slit) in both input and output surfaces, a resonant peak appearing at around $\Lambda$ emerges in the spectrum. This is a clear indication of the excitation by the incident atoms of a SMW running along the two interfaces. In a noncorrugated surface, due to momentum constraints, normal incident matter waves can only excite this SMW propagating from the slit toward the sides. The efficiency of this process is low, and the atoms coupled to the SMW are definitively lost for transmission because they travel away from the aperture. But a periodic array of grooves provides a momentum transfer of $\pm \hbar 2 \pi / \Lambda$, allowing the coupling of the impinging matter wave to the SMW. The coupling is more efficient when the number of grooves is increased, resulting in a higher transmission peak when $N$ goes from 5 (dash-dotted line) to 15 (dash-doubledotted line). In addition the transmission becomes spectrally narrower.

Another way to enhance the transmission through the slit is by tuning the value of the potential inside the grooves $\left(V_{\mathrm{g}}\right)$. This finding is illustrated in the right inset of Fig. 2. It shows how the transmission spectra for $N=10$ evolve as $V_{\mathrm{g}}$ is increased from $V_{\mathrm{g}}=V$ (as in the calculations presented in the main panel of Fig. 2) up to $V_{\mathrm{g}}=19 \mathrm{~V}$. There is an optimum value $V_{\mathrm{g}}=17 \mathrm{~V}$ for which the transmittance is maximum, resulting in an additional enhancement of 7 in $T$ with respect to the $V_{\mathrm{g}}=V$ case. It can be shown that this new mechanism to enhance the transmission through the subwavelength slit is related to the excitation of cavity modes inside the grooves,

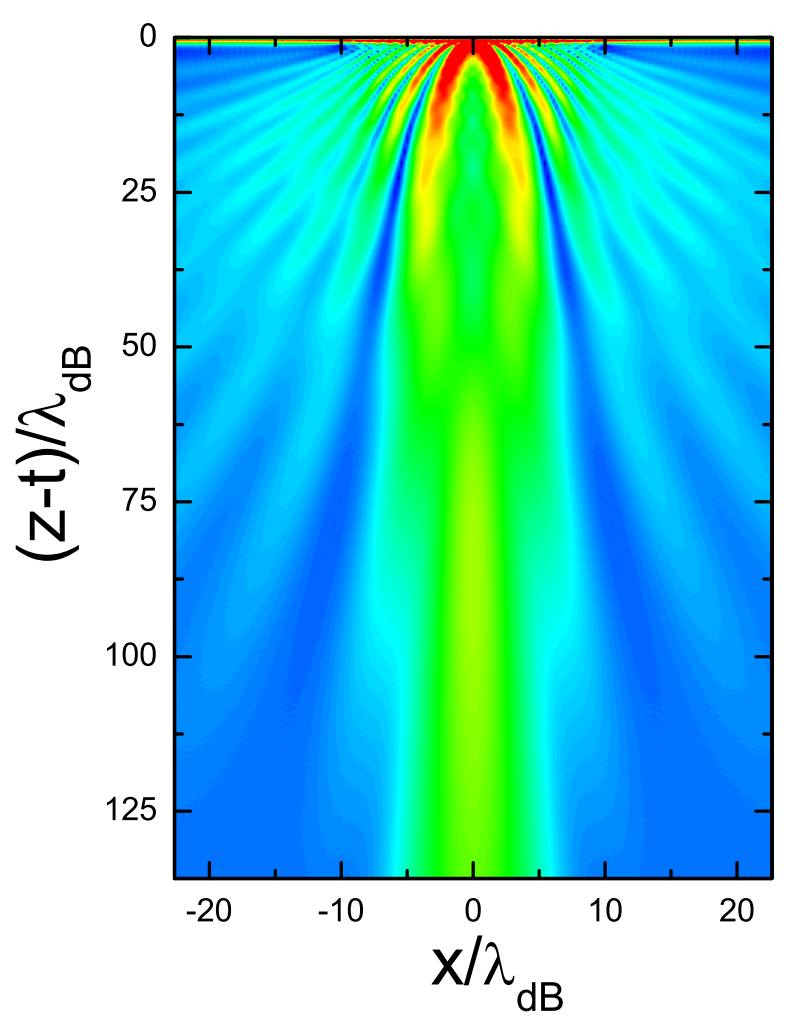

FIG. 4. (Color online) Modulus of the matter wave function for the case in which the central slit is surrounded by 20 grooves at the input and output surfaces of the structure. Potential inside the grooves, $V_{\mathrm{g}}=17 \mathrm{~V}$. The wave function is evaluated at the resonant de Broglie wavelength $\left(\lambda_{\mathrm{dB}}=1.1 \Lambda\right)$.

in a way very much similar to the mechanism found for light waves [7].

It is also worth commenting on the different roles played by the two interfaces in the transmission process. In the left inset of Fig. 2 we plot $T$ versus $\lambda_{\mathrm{dB}}$ for two cases: a single slit surrounded by $N=10$ grooves in both the input and output surfaces of the structure (solid line) and the same slit surrounded by $N=10$ grooves only in the input surface (dashed line). As clearly seen in this panel, the total transmission is basically controlled by the corrugation placed at the input surface.

Let us now analyze the beaming outside the aperture. The main panel of Fig. 3 renders the radial component of the current $\left(J_{r}\right)$ measured in the far-field region $(r=150 \Lambda)$ versus angle for the same four cases analyzed in Fig. 2. In all four cases $J_{r}$ is evaluated at the corresponding resonant $\lambda_{\mathrm{dB}}$ and is normalized to the total transmission (i.e., the integral of these four curves from $-90^{\circ}$ to $90^{\circ}$ is unity). For the single-slit case (dashed curve), $J_{r}$ is almost uniform as corresponds to a wave emerging from an aperture of width smaller than its wavelength. However, when an array of grooves is placed symmetrically at the output surface, the amplitude of the salient matter wave concentrates on $\theta=0^{\circ}$ producing a very collimated beam in the forward direction. Importantly, the divergence of this beam can be controlled by the number of grooves, decreasing as the number of grooves is increased. The same calculations have been done for structures corrugated only at the input surface. The results obtained are in- 
distinguishable from the single slit case. Therefore, we can safely conclude that the corrugation at the output surface absolutely controls the shape of the beam emerging from the structure.

The angular width of the beam can be further reduced by changing the potential inside the grooves. In the right inset of Fig. 3 we show how the beam width can be as small as $2^{\circ}$ for the case $V_{\mathrm{g}}=17 \mathrm{~V}$. The origin of this beaming phenomenon stems from the excitation of a running SMW on the output surface of the structure: this wave is a leaky wave since it emits matter radiation as it travels along the surface. A fingerprint of this leaky wave can be detected by analyzing the behavior of $J_{r}$ in the proximity of $90^{\circ}$ (see left inset of Fig. 3 ). This quantity gives an estimation of the amplitude of the leaky SMW after its passage through the finite array of grooves. Dashed curve renders $J_{r}$ for the single slit case whereas solid curve corresponds to the $N=10$ case. Clearly, the amplitude of the leaky SMW has been reduced. This decay is even more dramatic when $V_{\mathrm{g}}$ takes its optimum value ( $17 \mathrm{~V}$, dash-dotted line). The reduction of the amplitude of the leaky SMW reflects the fact that part of it has been radiated, achieving the above mentioned collimation effect.

To visualize how the beaming phenomenon takes place in this type of model structures, in Fig. 4 we plot the amplitude of the wave function (evaluated at resonant $\lambda_{\mathrm{dB}}$ ) in the output region $(z>t)$. Signals of the grooves emitting matter radiation are clearly seen in this picture. Interference of radiation coming from the central slit and the grooves $(N=10)$ that symmetrically flank it gives rise to the appearance of a collimated beam. It is also interesting to note the emergence of a very elongated focus centered at around $z=100 \lambda_{\mathrm{dB}}$ from the output surface, stressing the complexity in the behavior of the matter wave at the intermediate-field region (between near- and far-field regions). This focusing effect resembles the one observed in the photonic case $[13,14]$.

In conclusion, we have shown that the transmission of matter waves through a single subwavelength aperture can be enhanced when the aperture is flanked by a periodic array of indentations at the input surface. In addition, the cold atoms emerging at the other side can be collimated when the output surface is also corrugated. Both phenomena are due to resonant excitation of surface matter waves. These effects could find application in atom lithography [15] and interferometry [16].

This work was funded by the Spanish MCyT under Contract No. MAT2005-06608-C02 and the EC under STREP project Surface Plasmon Photonics (Grant No. FP6-NMP4CT-2003-505699).
[1] A. Landragin et al., Phys. Rev. Lett. 77, 1464 (1996). For a general review of the subject see Eur. Phys. J. D 35, 1 (2005), special issue.

[2] S. Marksteiner et al., Phys. Rev. A 50, 2680 (1994).

[3] V. I. Balykin, V. G. Minogin, and V. S. Letokhov, Rep. Prog. Phys. 63, 1429 (2000).

[4] T. W. Ebbesen et al., Nature (London) 391, 667 (1998).

[5] H. J. Lezec et al., Science 297, 820 (2002).

[6] L. Martín-Moreno et al., Phys. Rev. Lett. 86, 1114 (2001).

[7] F. J. García-Vidal et al., Phys. Rev. Lett. 90, 213901 (2003).

[8] L. Martín-Moreno et al., Phys. Rev. Lett. 90, 167401 (2003).
[9] E. Moreno et al., Phys. Rev. Lett. 95, 170406 (2005).

[10] R. J. Cook and R. K. Hill, Opt. Commun. 43, 258 (1982).

[11] J. P. Gordon and A. Ashkin, Phys. Rev. A 21, 1606 (1980).

[12] V. I. Balykin et al., Phys. Rev. Lett. 60, 2137 (1988).

[13] F. J. Garcia-Vidal et al., Appl. Phys. Lett. 83, 4500 (2003).

[14] E. Moreno, F. J. Garcia-Vidal, and L. Martin-Moreno, Phys. Rev. B 69, 121402(R) (2004).

[15] M. K. Oberthaler and T. Pfau, J. Phys.: Condens. Matter 15, R233 (2003).

[16] O. Carnal and J. Mlynek, Phys. Rev. Lett. 66, 2689 (1991). 